\title{
Obstructive sleep apnoea and depression: is there an association?
}

\author{
Edward O. Bixler, Jordan Gaines and Alexandros N. Vgontzas
}

Affiliation: Sleep Research and Treatment Center, Dept of Psychiatry, Penn State University College of Medicine, Hershey, PA, USA.

Correspondence: Edward O. Bixler, Sleep Research and Treatment Center, Dept of Psychiatry, Penn State University College of Medicine, 500 University Drive, Hershey, PA, 17033, USA. E-mail: ebixlerव pennstatehealth.psu.edu

@ERSpublications

Treatment strategies for OSA and depression need to be considered carefully as they share many common risk factors http://ow.ly/48v330bsm3y

Cite this article as: Bixler EO, Gaines J, Vgontzas AN. Obstructive sleep apnoea and depression: is there an association?. Eur Respir J 2017; 49: 1700858 [https://doi.org/10.1183/13993003.00858-2017].

In the study by KeNDZERSKA et al. [1] published in this issue of the European Respiratory Journal, it is reported that obstructive sleep apnoea (OSA) and severity measures, such as the apnoea/hypopnea index, are not related to the risk of hospitalisation for depression. The sample of the study was large with $>10000$ subjects, and the follow-up period was long, $\sim 10$ years. The investigators focused on the more severe phenotype of the disorder that requires inpatient treatment to avoid possible misdiagnosis, which is more likely in milder cases, due to overlapping symptoms between OSA and depression.

Early on, clinicians and researchers in the field of sleep medicine observed and reported a significant association between OSA and depressive symptomatology. In 1985, KALES et al. [2] reported high levels of psychological distress in a group of 50 patients with severe apnoea. Based on Minnesota Multiphasic Personality Inventory (MMPI) data, over half of the patients were seriously depressed and most of the symptoms focused on physical vulnerability. Furthermore, and based on MMPI profiles, the authors concluded that depression in OSA was a consequence, rather than a cause, of the disorder. Notably, in this study, there was no correction for important confounders such as obesity, a well-known risk factor for depression. Since then, most, but not all, studies in clinical and epidemiological samples have described a higher prevalence of depressive symptoms in patients with OSA compared with controls [3]. In a large community sample, $\sim 17 \%$ of patients with OSA had a major depressive disorder diagnosis, and even after controlling for obesity, the odds of having a breathing-related sleep disorder diagnosis (according to the Diagnostic and Statistical Manual of Mental Disorders, 4th Edition) were about five times higher for individuals with a major depressive disorder [4]. In clinical samples, the prevalence of depression in OSA patients ranges from $20 \%$ to $\sim 40 \%[3,5]$. Differences and inconsistencies among studies reflect methods of ascertaining OSA and depression, sample size and misclassification due to the overlapping symptoms of the two disorders.

There have been a few longitudinal studies, and the results are mixed. In 2006, PEPPARD et al. [6] studied participants in the Wisconsin Sleep Cohort, and reported a dose-response association between OSA and depression, suggesting a causal link between the two conditions. The diagnosis of depression was based on a structured questionnaire. However, the investigators did not control for weight gain, a known risk factor both for incident sleep disordered breathing and depression. Consequently, a population-based follow-up study from Taiwan reported that the incidence of depressive disorders was about twice as high among

Received: April 252017 | Accepted: April 292017

Conflict of interest: None declared.

Copyright OERS 2017 
patients with OSA compared with those without OSA [7]. However, this study was based on a database that included patients who had treatment for both OSA and depression, and thus a referral bias might have influenced the findings. More recently, amongst a representative sample taken from a large random general population sample from central Pennsylvania, LAGrotтe et al. [8] reported that obesity and excessive daytime sleepiness (EDS), but not OSA per se, are significant predictors of incident depression. The study was limited in that it did not contain severe cases most often found in clinical samples; the presence of depression was also based on self-report of physician diagnosis or treatment for depression. A novel finding of this study was the association of EDS with incident depression, a confounder overlooked in the previous studies.

In the study by KeNDZERSKa et al. [1], the risk factors for hospitalised depression were female sex, young age, hypnotic use, alcoholism and unemployment, all well-established risk factors for major depression. The authors appropriately caution the reader that their findings do not rule out the potential link between OSA and milder forms of depression. Despite the methodological differences and inconsistencies of the previously discussed studies, we can reasonably come to several conclusions. Depressive symptoms are more frequent in OSA patients, particularly in clinical samples. Severity of the disorder, obesity and EDS (frequently present in OSA patients) are strong risk factors for prevalent and incident depression. Current evidence, including the study by KENDZERSKA et al. [1], does not support a causal link between OSA and severe depression. Based on the clinical and psychological profiles of patients with OSA, it is likely that depressive symptoms are a consequence, rather than a cause, of the disorder.

OSA patients should be evaluated for depressive symptoms and appropriately treated, given the serious impact they have on the patients' lives, including family dynamics, social interactions and work situations. Treatment-resistant depressed patients with a risk profile for apnoea (i.e. those with obesity, EDS, hypertension, and/or diabetes) should be assessed clinically and, if indicated, in the sleep lab for the presence of OSA. Treatment of this disorder may improve their response to standard antidepressant therapy.

\section{References}

1 Kendzerska T, Gershon AS, Hawker GA, et al. Obstructive sleep apnoea is not a risk factor for incident hospitalised depression: a historical cohort study. Eur Respir J 2017; 49: 1601361.

2 Kales A, Caldwell AB, Cadieux RJ, et al. Severe obstructive sleep apnea--II: associated psychopathology and psychosocial consequences. J Chronic Dis 1985; 38: 427-434.

3 Harris M, Glozier N, Ratnavadivel R, et al. Obstructive sleep apnea and depression. Sleep Med Rev 2009; 13: 437-444.

4 Ohayon MM. The effects of breathing-related sleep disorders on mood disturbances in the general population. $J$ Clin Psychiatry 2003; 64: 1195-1200.

5 Basta M, Lin HM, Pejovic S, et al. Lack of regular exercise, depression, and degree of apnea are predictors of excessive daytime sleepiness in patients with sleep apnea: sex differences. J Clin Sleep Med 2008; 4: 19-25.

6 Peppard PE, Szklo-Coxe M, Hla KM, et al. Longitudinal association of sleep-related breathing disorder and depression. Arch Intern Med 2006; 166: 1709-1715.

7 Chen $\mathrm{YH}$, Keller JK, Kang JH, et al. Obstructive sleep apnea and the subsequent risk of depressive disorder: a population-based follow-up study. J Clin Sleep Med 2013; 9: 417-423.

8 LaGrotte C, Fernandez-Mendoza J, Calhoun SL, et al. The relative association of obstructive sleep apnea, obesity and excessive daytime sleepiness with incident depression: a longitudinal, population-based study. Int J Obes (Lond) 2016; 40: 1397-1404. 